\title{
Netzpolitik und Internet Governance
}

\subsection{Das Netz der Netze: Dimensionen seiner Steuerung}

Wie für alle Medien werden auch für das Internet Anforderungen und Bedarfe zur Regulierung gefordert oder für dringlich erachtet. Die Maßstäbe und Kriterien sind im Laufe der kurzen Geschichte des Internets von gut dreißig Jahren unterschiedlich ausgefallen, haben sich geändert und werden von verschiedenen Akteuren, mit diversen Interessen, Verbindlichkeitsgraden, mit unterschiedlichen Erfolgsaussichten und Durchsetzungsmöglichkeiten formuliert. Von den Nationalstaaten sind und werden diese Anforderungen meist ähnlich denen der konventionellen Media Governance vorgebracht; selbstverständlich sind sie vom Charakter des jeweiligen Regimes bestimmt - also von autoritär bis hin zu demokratisch-liberal und marktkonform libertär, oder wie sonst die typologischen Einordnungen lauten. Gemeinhin werden solche Entscheidungen und Regelungen als Netzpolitik bezeichnet (Donges 2007; Beckedahl und Lüke 2012); meist beziehen sie sich auf ein bestimmtes Thema oder Handlungsfeld mit spezifischen gesetzlichen Vorgaben (etwa für das Urheberrecht, den Datenschutz, die Verbreitung sanktionierter Inhalte etc.).

Die besondere Beschaffenheit des Internets - als Netz der Netze oder als Hyper-, Hybrid- und Transmedium (Bleicher 2010, 10) - treffen solche Maßnahmen allenfalls in Ansätzen, weshalb sie spezifisch und begrenzt sind, jeweils nachjustiert werden müssen oder gar scheitern, wie Kritiker, zumal engagierte User, häufig monieren. Allein schon der Singular der Bezeichnung ist irreführend. Denn das Netz ist kein einheitliches Medium, vielmehr eine komplexe, sich ständig 
erweiternde und ausdifferenzierende Infrastruktur. Sie ermöglicht diverse Dienste, die von verschiedenen Betreibern durchgeführt werden. Der Standard Transmission Control Protocol/Internet Protocol (TCP/IP) verbindet die verschiedenen Computer und Netzwerke miteinander. Erst durch diese Kombinationen wird Datenaustausch zwischen adressierten Computern, Netzwerken und Rechnern möglich (Seufert und Gundlach 2012, 286). Derzeit geht der Trend zu eher kleinräumigen Netzen (W-LAN), mit mobilen Geräten und mit Verlagerung von Betriebssystemen und Programmen in Netze (so genanntes Cloud Computing) und mit vielen flexiblen Anwendungsoptionen (Apps).

Außerdem erstrecken und realisieren sich die Netze jenseits aller nationalstaatlicher Hoheits- und Machtstrukturen; sie sind immer weniger territorial gebunden und statisch, sondern raumlos und fluid. Für die diversen Dienste - von der einfachen privaten Mail, über den Transport und die Verbreitung von massen- und crossmedialen Inhalten bis hin zu diversen, flexiblen Applikationen sind derzeit immer noch unterschiedliche Rechtskonstrukte zuständig, sofern sich die konvergierenden Optionen nicht gänzlich fixierten Standards und Regelungen entziehen. Allenfalls lassen sich einzelne Betreiber wie Provider, Suchmaschinen, Medienkonzerne und Diensteanbieter für die eine oder andere Handlungsdimension, den einen oder anderen Dienst- und Inhaltsbereich verantwortlich machen, nicht aber für das Netz als Ganzes. Deshalb forder(te)n Pioniere und engagierte User - zumal anfangs - das Netz möglichst frei von formellen, staatlichen und/oder internationalen Regelungen zu halten und höchstens Formen der Selbstregulierung der User und Beteiligten zu akzeptieren; zwischenzeitlich haben sich die Positionen und Forderungen diverser zivilgesellschaftlicher Gruppierungen weit ausdifferenziert, auch entsprechend ihrer (trans)kulturellen Ausrichtungen und ihres politischen Selbstverständnisses.

Auch viele Nationalstaaten zumal mit autoritären Regimen verstärken ihren Einfluss und streben nach Interventionen, wie die kursorische Übersicht zeigt (siehe Abschn. 5.2). So kursieren mittlerweile unendliche viele Standpunkte und Forderungen zum Internet bzw. zu einzelnen Sachverhalten zwischen den genannten Extremen, die schwerlich ganz überschaubar sind und auch häufig genug wechseln. Meist fokussieren sie sich auf bestimmte, jeweils dringliche Problem- und Handlungsfelder.

Anfangs und bisweilen bis heute werden nur technische Regelungen wie die Vergabe der Domain Namen, der Internet-Protokoll-Adressen (IP-Adressen) und die Betreuung der Root-Server-Systeme mit dem Terminus der Internet Governance belegt. Allerdings haben selbst in solch technische Regelungen gleich politische Interessen eingegriffen, so dass sie von medienpolitischen Zielen und Strategien nicht analytisch getrennt werden können. Wie die historische Übersicht (siehe Abschn. 4.2) 
zeigt, wurden und werden im Laufe der Entwicklung jeweils unterschiedliche Akzente gesetzt und sind verschiedene Akteure beteiligt. Daher bietet sich auch für das Hypermedium Internet an, den Governance-Begriff just für das komplexe System diverser Akteure und Interessen anzuwenden und ihn vor allem für verschiedene Phasen der Entwicklung offenzuhalten. So sah es schon eine ,Arbeitsdefinition' der Working Group on Internet Governance (WGIG) im Juni 2005 vor:

„Internet Governance is the development and application by Governments, the private sector and civil society, in their respective roles, of shared principles, norms, rules, decision-making procedures, and programmes that shape the evolution and use of the Internet" (Bandamutha 2010, 9).

Zuvor schon hatte J. Hofmann (2005, 10f) aus ihrer Rekonstruktion der Internet-Entwicklung (s.u.) die analytische Quintessenz einer noch breiteren Umschreibung gesucht, bei der unter Internet-Governance sowohl die sachlichobjektiven Prozesse als auch ihre analytische Reflexion umfasst werden: „Internet Governance [...] ist ein ergebnisoffener kollektiver Suchprozess, der darauf abzielt, eine globale regulatorische Leerstelle konzeptionell und institutionell in legitimer Weise zu füllen [...]. Die praktische Herausforderung des gegenwärtigen Suchprozesses im Bereich von Internet Governance besteht [...] darin, unter den Bedingungen von Transnationalität, partieller Deterritorialität und Dezentralität verbindliche und legitime Regelungskapazitäten für eine sich dynamisch entwickelnde Infrastruktur zu erzeugen."

All diese Dimensionen, Perspektiven, Erwartungen und Maßnahmen werden inzwischen mit dem Terminus Internet Governance umschrieben; deren Dimensionen lassen sich jeweils nur in den historischen Entwicklungsphasen, bezogen auf Handlungsfelder und Akteure, annähernd konkretisieren. Die folgende Übersicht soll zunächst exemplarisch veranschaulichen, welche Akteure bzw. Akteurstypen - ungeachtet ihrer tatsächlichen Einflussoptionen - möglicherweise in welchen Handlungsfeldern aktiv sind oder sein könnten (siehe auch: Donges und Puppies 2010, 87ff).

Je nach den Entwicklungsschüben von technisch-infrastrukturellen, medialkommunikativen, kulturellen, politischen und sozialen Faktoren ergeben sich Handlungsbedarfe und -positionen entweder kontinuierlich oder auch nur fallweise und zeitweilig; sie können sich modifizieren, wandeln oder ganz verschwinden. Die Zeitspannen werden immer kürzer, die Problemkomplexität eskaliert. Ihre Grundstrukturen und -probleme sollen in den folgenden Kapiteln dargestellt werden. Zur Gewinnung einer allmählichen systematischen Sichtweise sei daher zunächst knapp die historische Rekonstruktion der Organisationsentwicklung des Internets aufgezeigt, um daraus einige systematische Dimensionen $\mathrm{zu}$ gewinnen (Abb. 4.1). 


\section{Internet Governance}

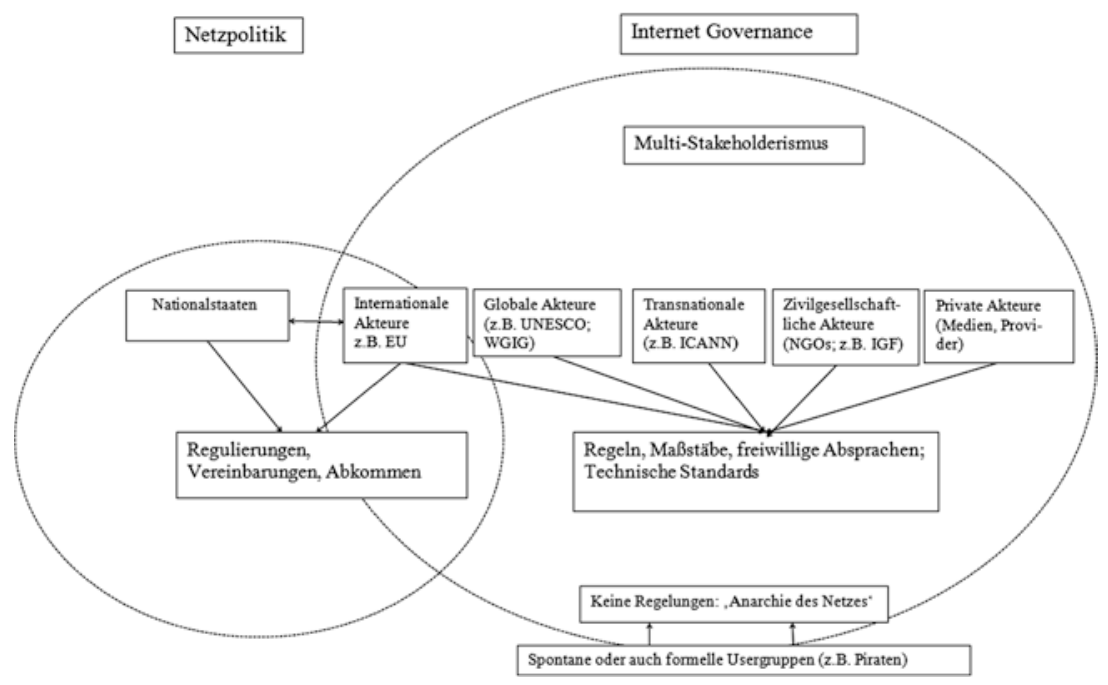

Abb. 4.1 Internet Governance (eigene Darstellung)

\subsection{Zur Geschichte von Internet Governance}

Die technische Geschichte des Internets und seine kommunikativ-medialen Entwicklungen sind schon vielseitig rekonstruiert und dargestellt worden; sie brauchen hier nicht erneut ausführlich rekapituliert zu werden (Gillies und Cailliau 2002; Mattelart 2003; Bleicher 2009; Stöcker 2011). Für die Thematik hier relevanter und aufschlussreicher ist die Rekonstruktion der gesellschaftlich-politischen Organisation des Internets, d. h. der Vorstöße und Modelle seiner Entwicklung wie seiner Regulierung. Diese spezielle Geschichte des Netzes, also der Konzepte und Maßnahmen zur Steuerung, Regelung, Nutzung und Bewertung des Internets nun als Internet Governance bezeichnet -, ist erst in Umrissen und mit recht unterschiedlichen Akzenten rekonstruiert. Denn diese Prozesse spielen sich - wie gezeigt - auf diversen politischen Ebenen ab und werden ebenso von verschiedenen Akteursgruppierungen realisiert. Immerhin zeichnen sich verschiedene Phasen ab, die sowohl mit den technischen Entwicklungen verzahnt sind als auch die unterschiedlichen Zwecksetzungen und Vergesellschaftungsgrade des Internets verkörpern: Dabei konkurrieren derzeit verschiedenartige Phaseneinteilungen und Typsierungen miteinander. Sie sind natürlich beeinflusst von den jeweiligen Absichten, die mit dem Internet verfolgt werden, sowie von den technisch-kommunikativen Potentialen, 
und sie lassen sich - je nach Perspektive - mit einem einigermaßen signifikanten Paradigma kennzeichnen und zeitlich voneinander abgrenzen. Wie bei allen historischen Einordnungen fallen sie umso willkürlicher oder auch beliebiger aus, je näher sie der Gegenwart kommen; erst mit angemessener Distanz dürften sie sich als markant oder gar typisch erweisen.

Im deutschsprachigen Raum haben sich besonders drei ExpertInnen mit der Entwicklung und den Strukturmerkmalen des Internets befasst, wobei nur Wolfgang Kleinwächter die Entwicklungen bis in die jüngste Gegenwart verfolgt und damit weit über eine nur historische Rekonstruktion hinausreicht, vielmehr aufschlussreiche Einordnungen der aktuellsten Prozesse und Entscheidungen liefert. Um ihre Sichtweise auf Geschichte, Gegenwart und Zukunft einschätzen zu können, seien sie kurz vorgestellt:

- Jeanette Hofmann $(2005 ; 2010)$ ist am Wissenschaftszentrum Berlin für Sozialforschung (WZN), in der Abteilung „Kulturelle Quellen von Neuheit“, tätig. Sie ist Gründungsmitglied und designierte Direktorin des in Gründung befindlichen und zunächst von Google finanzierten Forschungszentrums für Internet und Gesellschaft. Ferner ist sie Mitglied der Grünen Akademie der Böll-Stiftung und Sachverständige der Enquete-Kommission Internet und digitale Gesellschaft des Deutschen Bundestages und hat sich früh schon für ICANN engagiert.

- Wolfgang Kleinwächter (2009) ist Professor für Internetpolitik und -regulierung an der Universität Aarhus. Er war Mitglied der UN-Working Group on Internet Governance (WGIG) und leitete die Cross-Border Internet Expert Group des Europarates. Er veröffentlicht laufend Artikel vor allem in der Online-Zeitschrift Telepolis zur Entwicklung des Internets und zu den weltweiten Auseinandersetzungen um Internet Governance.

- Joan Bleicher ist Medienwissenschaftlerin an der Universität Hamburg und hat sich in zwei Publikationen (2009; 2010) mit der Geschichte und Ästhetik des Internets befasst.

\subsubsection{Initialphase und „technisches Regime“}

\subsubsection{Konzept- und Gründungsphase: das Internet als Technologie (1957-1970)}

Die Zeit zwischen 1957 und 1970 wird vorwiegend als die Phase der technischen Konzipierung und Gründung erachtet, für J. Hofmann (2005, 12ff) reicht diese Phase als „technisches Regime“ sogar bis 1995. Die 1950er und 1960er Jahre sind zugleich die des Kalten Krieges, also des meist technischen, mitunter auch 
militärischen Wettlaufs der Weltmächte USA und UdSSR bzw. des westlich-kapitalistischen und des östlich-(real)sozialistischen Systems um die Vorherrschaft, mindestens um die Beibehaltung ihrer Dominanz im eigenen Block. Für diesen Wettbewerb waren die Entwicklung und der geplante Ausbau eines angriffssicheren Netzwerkes vorrangig und dringlich. Konkret reagierte die USA auf das sowjetische Raumfahrtprojekt Sputnik spätestens seit dessen öffentlichen Start am 4. Oktober 1957 mit der Gründung der Advanced Research Projects Agency (ARPA), denn in der amerikanischen, aber auch in der weltweiten Öffentlichkeit wurde es als massive Bedrohung bewertet. In diesem Brain Trust sollten Wissenschaftler interdisziplinär mit Militärs Forschungen vorantreiben, unter anderem auch bei der Entwicklung von Netzwerken. Damit sollte nicht nur die militärische Abwehrfähigkeit, sondern auch der wissenschaftliche Vorsprung des Westens gefestigt werden. In Europa, etwa im Genfer CERN (Conseil Européen pour la Recherche Nucléaire), wurden mit dem CERNET ähnliche Konzepte des breiten, automatischen Datenaustausches in einem wissenschaftlichen Netzwerk verfolgt.

Zunächst wurde mit etlichen Netzen experimentiert, das Internet galt vor allem Experten aus der Telefonwelt als „akademisches Spielzeug“ (Hofmann 2005, 11). Deshalb verhielt sich die Privatindustrie zunächst recht skeptisch, während das Militär zunehmend den Nutzwert der Netzwerktechnologie für die Informationsvermittlung und militärische Kommunikation erkannte und seinen Einfluss verstärkte: 1969 entwickelten BBN Technologies das IMP (Interface Message Processor)-Netz, als Basis des so genannten ARPAnet (Advanced Research Projects Agency Network), und installierten es an verschiedenen Standorten. Es ist die Kerneinheit des heutigen Internets. Am ARPAnet waren seit 1969 auch etliche Universitäten beteiligt, etwa bei der Entwicklung von NetzwerkProtokollen sowie bei der Suche nach Anwendungsfeldern (z. B. Datentransfer, Telefon, Textübertragung, E-Mail). Um die militärische Relevanz dieser neuen Technologie zu demonstrieren, wurde Anfang der 1970er Jahre eine Verbindung zwischen der West- und der Ostküste in den USA eingerichtet, die dafür nötigen Instanzen zur Realisierung wurden öffentlichkeitswirksam als DARPA (Defence Advanced Research Projekt Agency) ausgerufen. Generell wuchs der militärische Einfluss, die Unabhängigkeit der Forschung schrumpfte, die Privatwirtschaft blieb abwartend. Aber schon damals entbrannte ein „Paradigmenstreit“ zwischen der Telefonwelt und der noch jungen Internetgemeinde über die Struktur und Kontrolle von Kommunikationsnetzen (Ebd.).

Mit der Entwicklung des einheitlichen Transmission Control Protocols (TCP) für internationale Kommunikation und Internetworking wurden internationale Absprachen erforderlich: Deshalb koordinierten sich europäische und amerikanische Vernetzungsprojekte als International Network Working Group (INWG). Um das 
Netz auch für private Geschäfte attraktiv zu machen, fehlten aber noch ebenso leistungsfähige Netze wie Computer (die Apple erst ab etwa 1977 bereitstellen konnte).

\subsubsection{Etablierung des Internets und Ausweitung der Dienste (1970-1989)}

Anfang der 1970er Jahre wurde das ARPAnet optimiert und als funktionsfähiges Netz installiert: Die Transferprotokolle wurden vereinheitlicht, die Dienste ausgeweitet - so erschien 1973 die erste Onlinezeitung, namens ARPAnet News - und der E-Mail-Verkehr nahm stark zu. Die DARPA beendete ihre spezielle militärwissenschaftliche Forschungstätigkeit und übergab ihr Netz dem US-Militär. Seit Juli 1977 wurde auch der militärische Datenverkehr über das ARPAnet erprobt, um so die Stabilität bei potentiellem Netzausfall durch atomare Schläge zu testen. Da die Privatwirtschaft allmählich das Geschäftspotential des Netzes, etwa für den E-Mail-Verkehr, erkannte, wurden Fragen der ökonomischen Verwertung, der öffentlichen Kontrolle sowie des militärischen Einsatzes verstärkt diskutiert. Sie wurden Ende der 1970er Jahre noch vorangetrieben, da erste Experimente mit grafischen Oberflächen - etwa an der Universität von North Carolina - bekannt wurden.

Nach wie vor waren das Internet und seine technischen Varianten sowohl innovatives Forschungs- und Experimentierfeld für viele Wissenschaftler und Technologen, effizientes oder zumindest erfolgversprechendes Instrument für den militärischen Datenverkehr als auch - allmählich - potentielles Geschäftsfeld für die Privatwirtschaft. Entsprechend überkreuzten sich die Anforderungen und Erwartungen, die Forschungskonzepte und Investitionen an und für das Netz, weshalb sie sich nicht einem homogenen Bestreben unterordnen lassen.

Gruppen aus eher wissenschaftlichen und zivilgesellschaftlichen Kontexten etwa die IEFT (Internet Engineering Task Force), eine informelle Gruppen von Ingenieuren - entwickelten schon in den 1980er Jahren Selbstorganisationsmodelle für das Internet, um möglichen staatlichen und intergouvernementalen Standardisierungen zuvorzukommen: „Governance without government" lautete die Maxime. Ein signifikantes Beispiel dafür ist die Netiquette - eine Sammlung von Richtlinien für faires Verhalten von individuellen Nutzern im Internet. In den sich mehrenden und grundsätzlicher werdenden Debatten schälten sich verschiedene Optionen für das Internet heraus: Einerseits wurde ihm das Potential zugeschrieben, Hierarchien und Monopole abzubauen, so mehr Partizipation für zivilgesellschaftliche Gruppierungen, aber auch für Individuen zu fördern, ja letztlich eine bislang unbekannte Freiheit in allen Kommunikationsformen zu erreichen; andererseits wurde die Gefahr einer wachsenden Unregierbarkeit von Staat und Markt beschworen (Leggewie und Maar 1998; Schröder 2012). 
In technischer Hinsicht wurden in den 1980er Jahre Fortschritte bei speziellen Netzwerken und Diensten erzielt, die allmählich das Internet formten: So konnte 1983 das erste Domain-Namen-System (DNS) eingeführt werden, um den Postverkehr zu optimieren. Weitere Software integrierte allmählich die traditionellen Massenmedien und Nachrichtenangebote und schuf damit die Basis für die seither beschworene Medienkonvergenz. Am 1. Januar 1983 wurde die zivile Nutzung des Internets eingeleitet: ARPAnet wurde nur noch für militärische Zwecke als Defence Data Network genutzt, bis zu seiner Einstellung 1990. Daneben entwickelten sich Kooperationsformen zwischen Wissenschaft und Wirtschaft; ihre Arbeiten zielten primär auf die Konzeption und Implementation einheitlicher Protokolle $\mathrm{ab}$, um den Datenaustausch zu vereinfachen und zu beschleunigen. Erst dadurch rückten lukrative Dienstleistungen (wie Shopping, Banking) ins Visier; dazu zählte auch die möglichst individuelle Vernetzung von Usern. Auch in wissenschaftlichen Kontexten wurden Fusionen vorangetrieben, so etwa die Vereinigung der wissenschaftlichen Netzwerke BITNET und CSNET in Großbritannien zur Corporation for Research and Educational Networking (NSFNET).

\subsubsection{WWW: Internet grafisch und kommunikativ (1989-1995)}

Mit der Entwicklung und Verbreitung des WWW (World Wide Web), konzipiert von Tim Berners-Lee im Genfer CERN, seit 1989, das eine einheitliche Schnittstelle für alle Informations- und Kommunikationsformen auf der Basis von Hypertexten ermöglicht, wurde das Internet grafisch und kommunikationsfähig; die Nutzerzahlen explodierten weltweit, nämlich Schätzungen zufolge von etwa 200.000 Anfang 1990 auf 9,5 Millionen (1996) und 72,4 Millionen im Jahr 2000 (Zimmer 2004, 2005, 169). Massenattraktiv wurde das neue Medium gegen 1993 mit dem Aufkommen von Internet-Browsern, die die bis dahin rein textlichen Navigationsoptionen durch grafische Oberflächen erweiterten. Angesichts solcher Verbreitungszahlen und Nutzungspotentiale entdeckte die Wirtschaft das neue Medium als vielfältige Plattform und künftig lukratives Geschäftsmodell.

In den USA wurden die staatlichen Subventionen der Infrastruktur allmählich reduziert. Die alten, eher internen Internet-Regeln von Wissenschaft und Zivilgesellschaft reichten bei fortschreitenden Kommerzialisierung und politischer Implementierung des Netzes nicht mehr aus. Die Debatte um angemessene Organisations- und Kooperationsformen - zunächst vor allem um die Vergabe von Domain Namen - begann, bald unter dem allgemeinen, aber vagen Begriff der Internet Governance. Daneben stellte sich mehr und mehr heraus, dass das generelle Postulat der Zugänglichkeit für alle nicht ohne zusätzliche, erhebliche Investitionen in die jeweiligen gesellschaftlichen Infrastrukturen verwirklicht 
werden könnte oder eben deklamatorisch bliebe: Das Schlagwort von „digital divide“ (digitale Spaltung) kam auf und verlangte nach politischen Antworten (siehe Abschn. 6.10). Aber zunächst fand sich kein prädestinierter Akteur oder wurde von der Netzgemeinde akzeptiert, weder nationale Regierungen noch intergouvernementale Organisationen wie die International Telecommunikation Union (ITU). Deshalb wurde weiterhin das libertäre, zivilgesellschaftliche Modell der Self-Governance favorisiert, mit Offenheit und Inklusivität, BottomUp-, Konsens-Orientierung und Dezentralität, das Netz gewissermaßen als Gegenkonzept zum nationalstaatlichen bzw. intergouvernementalen Typ des Regierens (Hofmann 2005, 16).

Dafür bildeten sich auch zusätzliche transnationale Organisationen, um unterschiedliche Interessen zu koordinieren: so 1992 die Internet Society, in der Unternehmen, aber auch Regierungen repräsentiert waren, oder zur Vereinheitlichung der Adressenvergabe 1993 das International Information Center (InterNIC), das regionale und nationale Kompetenzen delegierte, in Deutschland an das Deutsche Network Informationen Center (DENIC). In technischer Hinsicht machte die Entwicklung von Browsern Fortschritte, seit 1992 etwa Nescape, womit die Verbreitung des grafischen WWW optimiert wurde. Binnen drei Jahre errang der Netscape Navigator einen weltweiten Marktanteil von ca. 90 Prozent. Auch die Fusion mit den traditionellen Massenmedien und damit die Erweiterung des Internets zum publizistischen Medium ging voran: Am 25. Oktober 1994 eröffnete Der Spiegel als erstes deutsches Magazin seine Online-Version: spiegel.de (Kübler 2010, 98f). Es ist angeblich einige der wenigen Print-Expansionen, die Gewinne einfährt. Ebenso engagierten sich 1994, zum so errechneten 25-jährigen Jubiläum des Internets, zahlreiche Unternehmen und Institute dafür, verbindliche Umgangsregeln für Anbieter und Nutzer festzulegen, um so die ,Anarchie', das ,Bottum-up' von Entwicklern und Usern des Netzes der Gründerjahre, zu überwinden. Aber das wachsende ökonomische Potential des Internets beförderte unterschiedliche Geschäftsmodelle und rief weltweit agierende Medien- und Kommunikationskonzerne auf den Plan. So kollidierte die bislang geübte, informelle Praxis, die Domainnamen nach dem Prinzip „first come, first serve" zu vergeben, mit den wirtschaftlichen Interessen und der Regulierung von Warenzeichen (Donges und Puppis 2010, 85f).

\subsubsection{Institutionalisierung von Internet Governance (1995-2003)}

All diese Entwicklungen, aber auch Ansprüche verschärften gleichwohl den Druck, für das Internet Steuerungsmechanismen zu konzipieren und zu etablieren, die zwischen den verschiedenen Interessen und Akteuren Ausgleiche schaffen, 
mindestens Kernressourcen verbindlich zu regulieren vermochten. Zeitweise schienen dieses Bestreben einer effizienten Selbstregulierung sich auf ICANN (Internet Corporation for Assigned Names and Numbers), der privatrechtlichen Non-Profit-Instanz zur Registratur und Vergabe von Domain-Namen, zu fokussieren (Hamm und Machill 2001; Machill und Ahlert 2001). Sie wurde 1998 nach kalifornischem Recht in Los Angeles gegründet und bezog ihre Autorität aus der offiziellen Anerkennung durch das US-amerikanische Department of Commerce (DoC), also dem Handelsministerium. Zentrale Aufgaben der ICANN waren und sind großenteils noch immer die Verwaltung des Domainnamen-Systems (DNS), die Verteilung von IP-Adressen und die Koordination des Root-ServerSystems. Der enge Bezug zu den, wenn nicht die latente Dominanz der USA bei ICANN war von Anfang an Anstoß der Kritik aller weniger amerikafreundlicher Länder, aber auch aller zivilgesellschaftlichen Gruppen, denn das Prinzip der favorisierten transnationalen Self Governance wurde durch ein einseitiges Governance-Regime eines Nationalstaats („governance by government") unterhöhlt (Donges und Puppis 2010, 86). Dennoch galt ICANN zunächst als hochgelobtes Paradebeispiel für die Selbstorganisation des Netzes: Immerhin arbeiteten Vertreter der Privatwirtschaft und der Regierungen in einer eigens konstruierten Organisation zusammen; für die Nutzer war es das Modell einer transnationalen Nutzerorganisation, deren Partizipation 2000 in der weltweiten Online-Wahl des Direktoriums gipfelte. Doch nur etwa 170.000 User ließen sich registrieren, so dass die Wahl insgesamt die Vision zivilgesellschaftlichen Self Governance platzen ließ. Die Amtszeit der Gewählten endete 2002. Die dann erfolgte Reform verstärkte wiederum den Einfluss der nationalen Regierungen und schuf eine Art Drittelparität zwischen Wirtschaft, Technik und Zivilgesellschaft, die aber zunehmend zur privatrechtlichen Organisationsform tendierte.

In den letzten Jahren des 20. Jahrhunderts vollzogen sich viele technische und wirtschaftliche Entwicklungen, die das Internet in seinen basalen Strukturen bis heute kennzeichnen: 1998 wurde die MP 3-Technik für den Tausch von Musikdaten verfügbar; ein Jahr später kooperierten fünf führende Konzerne als MP 3-Association, um die Entwicklung des Audiokompressions-Standards voranzubringen. 1999 eröffnete die Tauschbörse Napster, die zunächst kostenlose Downloads anbot und Musik zum beliebtesten und billigsten Tauschartikel im Web machte. Ebenfalls 1998 startete die Suchmaschine Google mit etwa 320 Millionen Websites und wurde bald weltweit die alles beherrschende Such-Plattform mit diversen Offerten. Da das Ranking-System der Suche bis heute geheim gehalten wird, ranken sich um den verwendeten Suchalgorithmus, der auf der Zahl der Links basiert, viele Spekulationen, wodurch der globale Erfolg von Google eher noch gesteigert wurde (Lehmann und Schetsche 2005; Vise und Malsees 2006). 
Welche Erwartungen, wenn nicht überzogenen Phantasien sich an die ökonomische Potentiale des Internets knüpften, lässt sich daran ermessen, dass sich zur selben Zeit an den verführerische Slogan von der "New Economy“ viele grandiose Erwartungen knüpften: Digitalisierung und globale Vernetzung, neue Geschäftsideen und die Gründung vieler Startup-Firmen firmierten als Versprechen einer nunmehr virtuellen, nicht-materialen Wirtschaft. Zwei Jahre später krachten allerdings diese Visionen bereits an den Börsen und in den Märkten zusammen und intonierten das vorläufige Ende der New Economy. Kaum ein Jahr später wurde allerdings mit dem Web 2.0 als ebenso geschicktem PR-Slogan, also mit dem erweiterten, interaktiven und kommunikativen („Mit-Mach“-) Netz, die nächste Phase des Internets eingeläutet (Hass u. a. 2008; Ebersbach u. a. 2008).

\subsubsection{Intergouvernementale Organisationskonzepte: der anhaltende Kampf ums Internet (2003-2005)}

Mit seiner steigenden wirtschaftlichen und gesellschaftlichen Relevanz erwies sich das Internet als vielfältiges und immer wichtiger werdendes Handlungsfeld vieler internationaler, regionaler und auch nationalstaatlicher Instanzen. Längst sind die meisten von ihnen bei seiner Steuerung engagiert, suchen ihre angestammten Kompetenzen und Aufgaben zu behaupten und/oder streben danach, neue zu reklamieren bzw. sie anderen Akteuren abspenstig zu machen. Daher wachsen ständig Komplexität und Widersprüchlichkeit, überschneiden sich Interventionen und Ansprüche, übertrumpfen sich Positionen, Forderungen und Aktivitäten, so dass eher krasser Wildwuchs denn geordnete Konzepte von Internet Governance zu konstatieren sind.

Nach dem eher mäßigen Erfolg und der ambivalenten Resonanz der Aktivitäten von ICANN machten zwei spektakuläre Gipfel-Meetings zur Information Society (WSIS) aufmerksam, die die Vereinten Nationen über die UNESCO im Dezember 2003 in Genf und im Juli 2005 in Tunis als so genannte "Information Summits“ ausrichteten. Recht schnell brachen auf ihnen die Gegensätze zwischen den weniger entwickelten Ländern, denen sich die Weltmacht China als Fürsprecher andiente, und den westlichen Industrienationen darüber auf, welche Einflüsse und Regulierungskompetenzen die Nationalstaaten einerseits, die Wirtschaft, die zivilgesellschaftlichen Gruppen und der private Sektor insgesamt haben sollten. Die Entwicklungsländer favorisierten eher staatlichen Einfluss, der ihren Regierungen Regulierungsoptionen sicherte, während sich die Industrieländer für den so genannten „Multi-Stakeholder-Approach“ stark machten, also für das nur informell und situativ geklärte Konsortium von Wirtschaft, nationalen Regierungen, internationalen und zivilgesellschaftlichen Organisationen, bei dessen Handeln sich 
freilich gewissermaßen unter der Hand der jeweils Stärkere durchsetzte (Tharoor 2003; Dowe und Märker 2003). Im November 2004 setzte das Sekretariat der UNO eine Working Group on Internet Governance (WGIG) ein, die die politischen Sujets hinsichtlich des Internets herausarbeiten und Perspektiven für Regeln sowie Repräsentationen identifizieren sollte. Damit sollte der zweite Informationsgipfel im November 2005 in Tunis vorbereitet werden - wozu auch eine breite Definition von Internet Governance im Abschlussbericht vom Juli 2005 gehörte. Danach ist Internet Governance „the development and application by governments, the private sector and civil society, in their respective roles, of shared principles, norms, rules, decision-making procedures, and programmes that shape the evolution and use of the Internet" (zit. nach Kleinwächter 2009).

Noch erfolgloser als der erste verlief allerdings der 2. Informationsgipfel und bescherte vor allem den zahlreichen NGOs (Non Governmental Organizations) herbe Enttäuschungen. Immerhin verständigten sich die diversen teilnehmenden Gruppierungen auf eine Liste basaler Prinzipien für Internet Governance, darunter:

- "the recognition of national sovereignty over the ccTLD (country code toplevel domain), domain name space and the involvement of all stakeholders;

- the creation of an Internet Governance Forum (IGF) for five years

- the agreement on the launch of a process towards ,enhanced co-operation “" (Kleinwächter 2009).

Das IGF wurde als Ersatz für die (nicht erreichten) intergouvernementalen Strukturen vorgeschlagen, und zwar als Forum für alle beteiligten bzw. interessierten Stakeholders, ohne jegliche Entscheidungskompetenz. Es sollte fünf Jahre lang unter dem Schutz des UN-Generalsekretariats arbeiten und jährlich ein Meeting abhalten. Die Kontroverse um die Internet Governance konnte mithin nicht gelöst werden; sie wurde allenfalls vertagt (Hofmann 2005, 25). Als Extrempositionen stehen auf der eine Seite ein nur technisches Konzept, beschränkt auf die dringlich und alltäglich zu bewältigenden Standardisierungen (z. B. Domain-Verwaltung), auf der anderen eine sehr weite demokratische Interpretation mit allen politischen Implikationen wie Transparenz, Interessensausgleich, Behebung von Ungleichheiten („digital divide“), mithin eine gleichberechtigte, effiziente Steuerung weltweit (Marr und Zillien 2010; Stegbauer 2012). Dafür wurden über die Definition und Anerkennung allgemeiner Regeln hinaus konkret folgende Bereiche genannt:

- internationale Abkommen zu Handel und Wettbewerb

- Richtlinien zu Urheberschutz

- Datenschutz 
- Cybercrime

- oder auch nationale Maßnahmen zum Konsumentenschutz oder zur Regulierung der Telekommunikation (Hofmann 2005, 24).

Dagegen vertraten die neu entstehenden Internet-Mächte Russland und China (das 1998 ins World Wide Web aufbrach) betont nationale Interessen und suchten für ihren Politikansatz Verbündete. Im Abschlussbericht der Working Group on Internet Government (WGIG) vom Juli 2005 wurden gewissermaßen beiden Bestrebungen diplomatisch Rechnung getragen, denn einerseits wurden die allgemeinen Kriterien für die Governance-Strukturen wie Transparenz, Zurechenbarkeit und Demokratie betont, andererseits auch die nationalen und gouvernementalen Interessen berücksichtigt. So sollten

- staatliche Interventionen nicht umgehend und ausschließlich als Unterdrückung von Innovation und Kommunikationsfreiheit angesehen;

- über die Bedingungen und Optionen politischer Gestaltung des Internets nachgedacht;

- ein Problembewusstsein für die ungewollten Folgen einer grenzenlosen Kommunikation (z. B. Spams, Viren, Identitätsdiebstahl sowie andere Formen des Missbrauchs) entwickelt;

- die wachsende Verregelung von Datenverkehr und Kommunikationsinfrastruktur ständig kritisch überprüft;

- institutionell abgesicherte Beteiligungsformen etabliert werden, die es vermögen, ohne Rücksicht auf exklusive Souveränitätsprinzipien positive Wirkungszusammenhänge zwischen Legitimation und effektiver Problemlösungskapazität zu schaffen.

Denn übergreifendes Ziel für alle Beteiligten sollte es sein und werden, ein stabiles, allerseits anerkanntes Regelungsarrangement zu erreichen, das den wechselnden Problemlagen im transnationalen Datenverkehr wie auch den Beteiligungswünschen der interessierten Akteure in angemessener Form Rechnung tragen kann (Ebd, 26ff).

\subsubsection{Nationale und wirtschaftliche Einflüsse wachsen (2005-2012)}

Seit Mitte der ersten Dekade des neuen Jahrhunderts sind etliche Dienste auf dem Markt, die die Mitmach-Optionen des Web 2.0 vervielfältigen und verstärken: etwa seit 2004 die Möglichkeit, mit Podcast mobil zu empfangen, seit 2005 mit 
YouTube private Videos, inzwischen alle TV-Produkte umstandslos zu speichern und herunterzuladen, oder seit 2006 mit dem Microblog Twitter bis maximal 140 Zeichen Textnachrichten zu verbreiten und damit begrenzte Konkurrenz zu den professionellen Nachrichtendiensten und Medien zu ermöglichen. 2010 erreichte Facebook als Prototyp von Social Media nach eigenen Angaben 400 Millionen User weltweit. Weblogs soll es rund 200 Millionen geben (Bleicher 2010, 26ff).

Bei den Konferenzen des IGFs, im November 2006 in Athen und ein Jahr später in Rio de Janeiro, scheinen die Debatten um Internet Governance weniger kontrovers ausgefallen zu sein (Kleinwächter 2009); offenbar wurde der inzwischen eingeführte, so genannte „Multi-Stakeholder“-Mechanismus von allen Akteuren, den Nationalstaaten, der Industrie wie den Entwicklungsländern, den zivilgesellschaftlichen Organisationen sowie von der Wirtschaft und Industrie akzeptiert. Mit den USA wurde in Verträgen geregelt, dass der staatliche Einfluss auf ICANN reduziert wurde; 2009 wurde es als privates Unternehmen etabliert. Hingegen schlug die EU-Kommission im Mai dieses Jahres vor, die G 12-Staaten, also die wichtigsten Industrienationen und Wirtschaftsmächte, sollten mit dem Präsidenten von ICANN eine Art „Weltregierung des Internets“ bilden und über seine kritischen Ressourcen wachen (Kleinwächter 2009).

Seit 2010/11 wurde immer offensichtlicher, dass die schnell wachsenden Internet-Konzerne wie Google, Apple, Facebook und Amazon mehr und mehr auch die politischen Themen besetzen und dafür Strategien entwickeln, zumal die öffentlichen Akteure wie das IGF in Mandat und Auftrag eingeschränkt sind. Die künftigen Geschäftsfelder wie Netzneutralität, Cloud Computing und das so genannte "Internet der Dinge“ („thingternet“) dürften wohl nicht mehr ohne die Vorgaben und Interventionen der Konzerne geregelt werden. Auch die ehrwürdige International Telecommunication Union (ITU) - deutsch: Internationale Fernmeldeunion - entdeckte im Mai 2010 auf ihrer Konferenz in Mexico City das Internet als neues Regelungsterrain, zumal es zunehmend an die Stelle der terrestrischen Diffusion rückt. In Deutschland setzte der Bundestag eine neue EnqueteKommission mit dem Titel Internet und digitale Gesellschaft ein. Sie sollte schon bis Ende 2011 der praktischen Politik Vorschläge über Forschungsdesiderate und Regelungsbedarfe machen (http://www.bundestag.de/internetenquete). Erst im Laufe von 2012 wurden Zwischenberichte der einzelnen Fachkommissionen veröffentlicht, bei denen es im Kern auch weitgehend blieb. Im Januar 2013 folgte dann endlich ein eher deklamatorisches Abschlusscommuniqué ohne thematische Zusammenfassung, sondern mit persönlichen Einschätzungen der Mitglieder, in dem zumindest die Einrichtung eines ständigen Bundestags-Ausschusses vorgeschlagen wurde, da sich die Fraktionen bei den zentralen Fragen wie „Netzneutralität, Urheberrecht, Daten- und Verbraucherschutz sowie Green-IT“ [das ist 
ressourcen- und umweltschonender Gebrauch von IT-Geräten] nicht auf gemeinsame Beschlüsse und Empfehlungen einigen konnten. Im Wahljahr 2013 dürfte wohl keine der Handlungsempfehlungen politisch umgesetzt werden, und der neue Bundestag ab Oktober 2013 wird sicherlich seine politischen Prioritäten wieder neu setzen (Spiegel online, 26. Januar 2013).

Als weiteres Gremium wurde im Dezember 2010 die UN Working Group on IGF Improvement eingesetzt, und zwar nach einigen Querelen mit 23 Vertretern von Regierungen, jeweils fünf der Privatwirtschaft, der Zivilgesellschaft, der technischen und akademischen Community sowie von zwischenstaatlichen Organisationen. Da die Zukunft des IGF ungewiss war, sollte diese Working Group weitere Vorschläge für Internet Governance ausarbeiten. Auch die Leitung von ICANN geriet immer wieder in Kontroversen, da insbesondere neue, meist auch ideologisch ausgerichtete Internet-Staaten wie Iran und Saudi-Arabien ihren Einfluss stärken wollen; sie werden von China unterstützt, das weiterhin eine Renationalisierung des Internets weltweit anstrebt und diese Strategie in seinem Territorium bereits weitgehend durchgesetzt hat. Brasilien, Indien und Südafrika schlugen hingegen eine neue zwischenstaatliche Plattform vor, die den „Multi-Stakeholder“-Prozess ergänzen, aber nicht ersetzen sollte. Auf der 66. UN-Vollversammlung im Oktober 2011 wurde dieser Streit erneut ausgetragen, der besonders dann aufbricht, wenn Ergänzungen oder Optimierungen der Aufgabenfelder anstehen. Inzwischen ist das Mandat von ICANN bis 2015 fixiert, der ,Multi-Stakeholderismus' soll für diese Aufgaben weiter entwickelt werden (Kleinwächter 2010, 2011a, b, c, 2012).

Auch der G 8-Gipfel, die kleinere Runde der Weltwirtschaftsnationen, befasste sich Ende Mai 2011 auf Vorschlag des Gast gebenden Frankreichs mit „eG 8-Cybersecurity“. Inzwischen kümmern sich fast alle internationalen Organisationen und Instanzen (siehe Abschn. 5.2) um das Internet bzw. um Internet Governance, entsprechend ihrer jeweiligen Aufgabenperspektiven und Interessen: etwa

- die UNO mit IGF (Internet Governance Forum) und WSIS, der weiter bestehenden Organisation für die UN-Weltgipfel zur Informationsgesellschaft, hinsichtlich allgemeiner, globaler Regelungen für das Internet,

- die NATO mit militärischen Sicherheitskonzepten und mit einem speziellen Sicherheitszentrum in Estland, das Committee for Information, Computer and Communication Policy (ICCP),

- die OECD vor allem zu wirtschaftspolitischen Optionen des Netzes,

- der Europarat mit seiner Cross Border Internet Expert Group in einer im Juni 2011 veröffentlichten Deklaration zu Menschenrechten, „Multi-Stakeholderism“, offenes Internet, kulturelle Vielfalt und Sicherheit, 
- die OSZE mit zwei Internet-Konferenzen 2011 in Tiflis und Duschambe, um ebenfalls ihr Internet-Engagement zu demonstrieren,

- die ITU mit einem Forum im Mai 2012, um ihrer Rivalität zu ICANN über die Kontrolle der kritischen Internet-Ressourcen zu bekräftigen.

Auch kontinental, regional und infolge übereinstimmender Interessen schließen sich Staaten und Akteure zusammen. So gibt es regionale IGFs auf allen Kontinenten, die ihre Konferenzen veranstalten und Deklarationen verabschieden. Im September 2011 schlugen die IBSA-Staaten - das sind Indien, Brasilien und Südafrika - eine neue zwischenstaatliche Internet-Organisation bei der UNO vor. Sie wie die so genannte Shanghai-Gruppe mit China und Russland als Wortführer brachten sowohl auf der 66. UN-Vollversammlung 2011 als auch auf der folgenden 67. im Jahr 2012 Internet-Verhaltenskodizes sowie die Zukunft des IGFs auf die Tagesordnung; bislang mit wenig definitiven und konsentierten Erfolg.

Auch der vom britischen Außenminister William Hague 2011 initiierte, so genannte „Londoner Prozess“ zur Ausarbeitung globaler Internet-Prinzipien zeitigte auf den zwei bislang abgehaltenen, hochrangig besetzten Konferenzen kein greifbares Ergebnis. Ebenso wurde auf der Münchner Sicherheitskonferenz im Januar 2011 erstmals das Thema Sicherheit im Cyberspace diskutiert und daraus gefolgert, neben den supra- und internationalen Gremien auch nationale Positionen und Instanzen einzubeziehen. Doch der institutionelle Wirrwarr und die strategischen Konkurrenzen werden durch die Vielzahl von Akteuren gewiss nicht kleiner und effizienter. Lediglich markiert diese Tendenz, dass Regelungen und Sicherheitsstandards inzwischen zunehmend auch als dringliche Aufgaben der nationalen Außenund Sicherheitspolitik betrachtet werden, mithin eine Renationalisierung der Internetpolitik ansteht, so wie es die neuen Internet-Mächte - allen voran China, Russland und Iran, aber auch Indien, Frankreich, Polen, Brasilien, Südafrika, SaudiArabien u. a. - mit je verschieden gewichteten und artikulierten Interessen anstreben, und dass ursprüngliche Visionen, das Internet könne nationale Ansprüche überwinden und in transnationaler Governance funktionieren, weit zurückgedrängt sind.

Damit gerät das Internet auch zwischen die bestehenden realen Fronten und Konkurrenzen der (National)Staaten, wie sie erneut bei der World Conference on International Telecommunications (WCIT-12) der ITU im Dezember 2012 in Dubai aufbrachen. Für eine Neufassung ihrer Aufgaben und Regeln, die noch von 1988 stammen und natürlich nicht die aktuelle Situation des weltweiten Datenverkehrs erfassen, schlugen arabische und afrikanische Staaten vor, dass die Regierungen volle Kontrolle über IP-Adressen und Domainnamen haben sollten. Gleichzeitig sollten sie die Möglichkeit bekommen, jeden Internetnutzer zu identifizieren. Doch wieder einmal konnten die westlichen Internet-Mächte, allen 
voran die USA, solche Interventionsabsichten verhindern, so dass es bis auf weiteres bei den nicht mehr zeitgemäßen Regeln bleibt (Kleinwächter 2012b, 2012c).

Insgesamt waren für das Jahr 2012 mehr als 50 „very important meetings“ zu Internet Governance mit dem Ziel zu verzeichnen, allseits anerkannte Regelungen zu treffen, individuelle Freiheit, wirtschaftliches Wachstum und soziale Entwicklung gleichermaßen zu fördern. Allein das relativ selbständige, nur an die UN angebundene IGF ist nach Einschätzung von W. Kleinwächter (2012a) in der Lage, ein universelles, potentiell von vielen akzeptables, aber wohl recht unverbindliches Dokument ein Multistakeholder Framework of Commitment for Internet Freedom - womöglich in den nächsten Jahren (bis 2015 oder 2016), auf den bereits wieder zahlreich anberaumten Konferenzen, zu erstellen - sofern es dann noch erforderlich ist und nachgefragt wird. Davor wird die UNESCO bis 2015 die Resultate ihrer Informationsgipfel überprüfen, ITU und ICANN könnten ihren „kalten Frieden“ in konstruktive Bahnen lenken, die UNO-Vollversammlung Positionen zum Management kritischer Internet-Ressourcen und zur so genannten „enhanced co-operation“ erarbeiten sowie alle anderen Gremien ihre Interessen und Aufgaben kooperativ definieren. Oder aber die hartnäckigen, allgemeinen politischen und ideologischen Gegensätze beherrschen auch weiterhin die Internet Governance: Grob polarisiert, behaupten sich auf der einen Seite die etatistischen oder gar autoritären Strategien, die das Internet wie alle anderen Medien zunächst einer nationalen staatlichen Kontrolle unterwerfen (wollen) und international allenfalls eine zentralisierte zwischenstaatliche, mit starker Regierungsbeteiligung versehene Organisation hinnehmen, die für das Internet als Ganzes zuständig ist und eine „Kontrolle von oben“ installiert. Ihnen konträr sind - allerdings ebenfalls verschiedene - dezentral funktionierende Modelle von „Multi-Stakeholder“-Governance, bei denen Regierungen, Privatwirtschaft, Zivilgesellschaft und technische Community in ihren jeweiligen Rollen gleichberechtigt zusammenarbeiten, das Netz der Netze weiter entwickeln und diversifizieren, um die Selbst-Regulierungskräfte und wechselseitigen Verantwortlichkeiten aller Betroffenen und Beteiligten zu stärken (Ebd.). Natürlich ergeben sich viele Konzepte dazwischen, je bestimmt von den Managementstrukturen, Beteiligungsproportionen, Aufgabendefinitionen und Interessendominanz etc. - weshalb es wohl kaum zu einheitlichen, allseits akzeptierten Generallösungen kommen wird, sondern immer wieder vielfältige partielle und pragmatische Kompromisse gesucht werden müssen.

\subsubsection{Ein strukturelles Fazit aus der Retrospektive}

Überblickt man die verschiedenen Phasen und unterschiedlichen Schwerpunkte dieser - hier nur kursorische - Rekonstruktionen, lässt sich erkennen, dass für 
so komplexe, auch diffuse Entwicklungen wie die der Internet Governance noch keine konsensfähigen Auswahl- und Konstitutionskriterien vorliegen, sofern sie überhaupt je möglich sind bzw. werden. Allenfalls lassen sich einige grobe Gewichtungen beschreiben:

1. In der ersten Phase herrscht zunächst eine eher privatwirtschaftliche oder wissenschaftspolitische Governance der noch ganz unterschiedlichen InternetInnovationen und -varianten vor.

2. In der zweiten Phase nehmen militärische Interessen überhand (ARPAnet), ohne dass klar abgrenzbar ist, wie diese bei den weiteren Entwicklungen von privatwirtschaftlichen und wissenschaftspolitischen Einflüssen überlagert werden oder mit ihnen korrespondiert haben.

3. Danach folgt die (dritte) Phase der libertären Gründerzeit des Internets, in der es vornehmlich Experimentier- und Innovationssektor für technische und wissenschaftliche Akteure gewesen ist. In ihr überbieten sich Visionen von einem freien, für jede/n zugänglich, von jedem/r entwickelbaren Netz gewissermaßen als erstes anarchisches Kommunikationsmittel, von einer neuen elektronischen Grass-Root-Demokratie oder einem Athenischen Zeitalter (US-Vizepräsident Al Gore). Regelungen trifft die Netz-Community autonom oder - wenn es Institutionalisierungen bedarf - von ihr beschickte Gremien, wie es zeitweise für ICANN vorgesehen war.

4. Diese für die einen neu- und großartigen, für die anderen utopischen, wenn nicht idealistischen Modelle von Internet Governance sehen sich mit der Entwicklung und Verbreitung des World Wide Web sowohl mit wachsenden kommerziellen als auch mit politischen Interessen und Anwendungen konfrontiert, die von den unterschiedlichen Regimen der Nationalstaaten vertreten wurden und werden. Darüber hinaus avanciert das Internet zum internationalen Politikum, besonders in seinen verteilungspolitischen Folgen, die sich auf Reizthemen wie „digital divide“ und Netzneutralität kaprizieren. Vor allem supranationale Organisationen wie die UNESCO entdecken das Internet als neues, auch entwicklungspolitisches Betätigungsfeld, wie die beiden Information Summits 2003 (Genf) und 2005 (Tunis) ausweisen. Dabei sollten alle Governance-Akteure und -Interessen in weltweite Absprachen und Maßnahmen einbezogen werden, um so Interessensausgleiche zu schaffen.

5. Da das Internet inzwischen auch zur politischen Waffe für Oppositionen und Attacken zumal in und gegen autoritäre Regime geworden ist, sind jene Konzepte zum Scheitern verurteilt und behaupten sich nationalstaatliche Regulierungen bis hin zu strukturellen und permanenten Zensurmaßnahmen (wie etwa in der VR China). Damit ist diese vierte Phase eher von Renationalisierungsabsichten 
bestimmt, die das Internet in die allgemeinen Konflikte und Konkurrenzen (wieder) einbezieht und es zum Spielball der jeweils herrschenden Regimen und Regierungen macht. Ob solche Strategien auf Dauer gelingen oder sie von kompetenten Usern im aterritorialen, transnationalen Netz infolge seiner technischen Struktur, seiner kommunikativen und wirtschaftlichen Potentiale immer wieder unterlaufen werden können, wird sicherlich kontrovers gesehen, aber auch künftig die Regulierungs- oder Deliberationspolitik bestimmen.

6. Gegenwärtig sind die Konferenzen, Konzepte und Maßnahmenvorschläge von ganz verschiedenen Akteuren kaum mehr überschaubar; sie fallen zudem für die diversen Handlungsfelder - wie sie unten heuristisch beschrieben werden - ganz unterschiedlich aus. Einerseits beschäftigen sich nahezu alle supranationalen Organisationen mit Dimensionen des Internets, zum anderen befassen sich die Staaten entsprechend ihrer ideologischen Konzepte mit ihm bzw. mit Regulierungsbereichen, die auch außerhalb des Internets - wie z. B. Technologie- und Wirtschaftsförderung oder Marktregulierung - bereits bestehen und die sich mit der digitalen Welt erweitern oder neu ergeben. Gewissermaßen hat das Internet eine Reintegration von Internet Governance in die konventionellen Politikfelder erfahren. Einerseits werden diese Prozesse als Renationalisierung von Internet Governance bezeichnet, was den gesamten Sachverhalt mit seinen globalen Komponenten nicht ganz trifft; treffender ist andererseits die Perspektive, dass die Exklusivität und gänzliche Alternativität des Internets erodiert und eine Art ,Normalisierung von Internet Governance mindestens über die diversen Handlungsfelder stattfindet.

Für die weitere Entwicklung des Internets und der Internet Governance stellte Anfang 2012 W. Kleinwächter (2012a) folgende Fragen:

1. „Wie finden wir auf globaler Ebene ein neues Gleichgewicht zwischen in Konflikt zueinander stehenden Werten wie Sicherheit, Eigentum, Freiheit und Privatsphäre unter Berücksichtigung der verschiedenen politischen Systeme, der unterschiedlichen Kulturen, Traditionen und historischen Erfahrungen?

2. Wie entwickeln wir ein neues Verhältnis zwischen staatlichen und nichtstaatlichen Institutionen, die nicht auf einer hierarchischen Unterordnung basieren, sondern auf gegenseitiger Zusammenarbeit unter Gleichen entsprechend der spezifischen Rollen und Verantwortlichkeiten der einzelnen Akteure?

3. Wie verbinden wir traditionelle zentrale, geschlossene und Top-downPolitiken von Regierungen mit den offenen, transparenten und Bottom-upMechanismen, die typisch sind für die Internetwelt?“

Die Beantwortung und Klärung dieser Fragen dürfte sicherlich über die Zukunft von Internet Governance (mit)entscheiden. 


\subsection{Inoffizielle Internet Governance durch Internet-Konzerne}

Wenn Governance mehr als die intentionale, womöglich gesetzliche und/oder vertraglich legitimierte Setzung allgemeiner Regeln durch identifizierbare, verantwortliche Akteure umfasst, nämlich etwa funktionale Formierungen von Prozessen und Handlungen, die durch ihre systemische und habituelle Internalisierung selbstverständlich, quasi technikbedingt sind, dann muss mit einem solch weiten Ansatz auch untersucht werden, welche Anteile und welche Methoden InternetKonzerne an einer solchen inoffiziellen oder latenten Governance haben. Darüber wird bereits unentwegt in Medienkritik und Feuilleton spekuliert und räsoniert, wissenschaftlich-analytisch fundierte Befunde gibt es hingegen noch vergleichsweise wenige. Mithin sind die Abgrenzungen und Identifizierungen zwischen analytischen und nur heuristischen, interpretativen Zugängen schwierig zu markieren.

Exemplarisch lassen sich solche Fragen etwa auf die „Big Four“ der gegenwärtigen Internet-Ära fokussieren, also auf Google, Apple, Facebook und Amazon (Bethge u. a. 2011). Das Nachrichtenmagazin Der Spiegel charakterisierte in seiner Titelgeschichte „Web-Kampf um die Zukunft. Wer beherrscht das Internet?“ im Dezember 2011 jeden der vier Web-Konzerne, der „Supermächte des digitalen Lebens" (Ebd., S. 72), die gegenwärtig den ökonomischen und kulturellen „Führungsanspruch“ der USA in der Welt verkörpern, mit einem paradigmatischen Ideal, nämlich mit dem

- „Ideal totalen Wissens“: Google offeriert, selegiert und bewertet das Wissen der Welt und gibt seine so latent getroffene Auswahl als scheinbar umfassend, total und objektiv aus; damit steuert und gewichtet der Konzern Information und Wissen in der Welt;

- „Ideal totaler Mobilität“: Apples iPod, iPhone, iPad und mit den auf ihnen ladbaren Apps ermöglichen und suggerieren die völlige Allgegenwärtigkeit digitaler Kommunikation und Erreichbarkeit, generieren somit einen totalen virtuellen Kosmos, ,in dem alles transferierbar, synchronisierbar, herunterladbar, tragbar, übersetzbar sein soll“ (Ebd., 74f). Damit sinkt der Bezug zur physischen und sozialen Wirklichkeit, was womöglich nicht ohne Einfluss auf die Wahrnehmung, Erfahrung und Vorstellung von Welt überhaupt bleibt;

- „Ideal totaler Transparenz": Mit weltweit über 900 Millionen User (Mai 2012) organisiert das soziale Netzwerk Facebook den größten „Verein“ der Welt vorderhand als persönliche Freundschafts- und Neigungskreise („Gefällt-mir“Button bzw. „likes") und individuelle Selbstdarstellungen besonders durch die seit 2012 eingeführte „timeline-Funktion“, tatsächlich als mächtigster Sammler 
und Verkäufer persönlicher Daten, der längst schon soziales Zusammenleben, interindividuelle Wahrnehmung und Modalitäten persönlicher Interaktivität nach seinen formalen Vorgaben steuert. Für Datenschützer ein Alptraum, ist es offenbar für Millionen von Usern noch immer chic, bequem und zeitgemäß, ihre gesamte Privatheit im Netz zu offenbaren und damit Industrie und Werbewirtschaft ständig präziseres Profil zu liefern;

- „Ideal totaler Verfügbarkeit“: Amazon ist nicht nur das größte OnlineKaufhaus der Welt und suggeriert die „totale Verfügbarkeit aller Waren und Genüsse der Welt“ (Ebd.); mit ständig akkurateren Identifikationstools rückt es dem einzelnen Kunden immer enger auf dessen Identität und bietet ihm je spezielle und individuelle Angebote für sämtliche Bedürfnisse. Darüber hinaus scheint Amazon mit seinem E-Book „Kindle“ (und all den anderen Varianten) das Buchlesen, wie es sich seit Erfindung des Buchdrucks allmählich herausgebildet hat, zu verändern, womöglich zu revolutionieren: Nicht nur, dass man seine Hausbibliothek quasi digital verfügbar hat - freilich bislang nur Titel, die aus ökonomischer Sicht vom jeweiligen Provider online zur Verfügung gestellt werden -, auch die sinnliche Erfahrung des gedruckten Buches scheint verloren zu gehen. Hingegen gewinnt man Optionen des alinearen, assoziativen Lesens (mit Mehrwert-Funktionen hin zu zusätzlichen Informationen und Texten) sowie des „social readings“, wenn man per Internet mit anderen Lesern korrespondieren kann.

Sicher handelt es sich bei diesen Porträts um journalistisch motivierte, recht plakative Zuspitzungen, die sich außerdem infolge spezieller Entwicklungen modifizieren oder gar erledigen können. Daher dürften sich diese „Ideale“ sicherlich ständig modifizieren und gegenseitig beeinflussen, zumal die vier Megakonzerne sich offenbar anschicken, sich ihre Geschäftsterrains streitig zu machen und für ihre Kunden jeweils alle Funktionen anzubieten. Gleichwohl weisen die Kennzeichnungen darauf hin, dass infolge der Komplexität und Interdependenz der Sachverhalte breit formierende, gar systemische Konsequenzen unausweichlich sind, die sich wie Entscheidungen und Maßnahmen einer inoffiziellen Governance auswirken.

Anlässlich der Vorgänge und Debatten um die für 2014 vorgesehene DatenschutzGrundverordnung der Europäischen Union ist Ende 2012 und Anfang 2013 bekannt geworden, dass und wie mächtig sich die globalen Internet-Konzerne wie Microsoft, Google, Facebook und andere in die gesetzlichen Formulierungen einmischen und wie sehr EU-Administration und Abgeordnete des Europäischen Parlaments von der vehementen Lobbyarbeit beeinflusst sind. Unverfroren wird mit der Aufkündigung der bestehenden (Gratis)Geschäftsmodelle und der Verteuerung 
der Angebote gedroht, wenn die EU-Verordnung zum Datenschutz weitgehend dem deutschen Datenschutzrecht gleiche. Daher zielt die Lobbyarbeit auf eine unternehmensfreundlichere Fassung ab, die Eigenverpflichtungen und Selbstkontrollen der Unternehmen bevorzugt und damit die vorliegende Version deutlich abschwäche, sei es bei der Einwilligung in die Datenverarbeitung oder bei den Meldepflichten im Falle einer Datenpanne. Horrend haben die IT-Konzerne dafür ihre Aufwendungen für Lobbyarbeit in den USA und in Europa angehoben: So hat Google im Jahr 2012 mehr als 16 Millionen Dollar für seine Lobbytätigkeit in den USA ausgegeben, 70 Prozent mehr als im Vorjahr. Facebook investierte knapp vier Millionen Dollar, was einer Steigerung um 196 Prozent im Vergleich zum Vorjahr entspricht. Microsoft gab gut acht Millionen Dollar aus, rund zehn Prozent mehr als noch im Jahr 2011. Bei Amazon waren es 2,5 Millionen Dollar (http://www.zeit.de/digital/ datenschutz/2013-01/datenschutztag-datenschutzverordnung-lobbyismus).

Gegenüber solcher eher noch dem überkommenen Politikverständnis entsprechenden Strategien setzen tiefer greifende Analysen an, die etwa am Beispiel des dominanten Marktführers Google untersuchen, wie Suchmaschinen Beherrschungsbestrebungen und Selektionsentscheidungen als eigentlich „undurchschaubare, orakelhafte Algorithmen“, mithin als vermeintlich technisch und logisch bedingte Rationalitäten verklären, "die sich nicht auf eine eindeutig verortbare Intentionalität zurückführen lassen" (Röhle 2010, 14ff).

Schon der innovative PageRanking-Algorithmus firmiert bis dato als bestgehütetes Geheimnis des Geschäftsmodells (Vise und Malseed 2006, 58ff), den viele der Millionen Nutzer kaum durchschauen und bei ihrer Suche vergegenwärtigen. Doch auch Suchexperten kapitulieren: Denn der Kern der Software für Googles Suchmaschine umfasst Hundertausende Code-Zeilen. Mit ihm wird die Linkpopularität einer Seite bzw. eines Dokumentes festgelegt. Das Ziel des Verfahrens ist es, die Links dem Gewicht entsprechend zu sortieren, um so eine Ergebnisreihenfolge bei einer Suchabfrage herzustellen, d. h. Links zu wichtigeren Seiten weiter vorne in der Ergebnisliste anzuzeigen. Sein Grundprinzip lautet: Je mehr Links auf eine Seite verweisen, umso höher ist die Relevanz dieser Seite. Und je höher die Relevanz der verweisenden Seite ist, desto größer ist der Effekt. Je komplexer und ,intelligenter' die Systeme werden, desto schwieriger wird diese Gewichtung nachzuprüfen sein, nicht zuletzt auch für Google-Mitarbeiter selbst, die „selbst nicht genau wissen, was warum funktioniert", und nur noch das Ergebnis sehen (Pariser 2012, 212).

Die Chance, möglichst oft und prominent gefunden zu werden, zahlt sich zwischenzeitlich als Marketingstrategie und womöglich steigenden Umsatz pekuniär aus. Sie befördert daher viele gezielte Manipulationen wie Spamming in Gästebüchern, Blogs und Foren, Betreiben von Linkfarmen und andere 
unseriöse Methoden. Auch die Usance, den in der Toolbar angezeigten PageRank einer niedrig eingestuften Seite durch Weiterleitung auf eine bestehende Seite mit hohem PageRank zu spiegeln, gehört dazu. Denn das Weiterleiten bewirkt, dass die Anzeige des hohen PageRanks der Zielseite mit dem folgenden Update kopiert wird. Nach wie vor ranken sich daher viele Mythen und Legenden um das PageRanking; doch inzwischen werden sie fast völlig von den vermeintlichen Zweckrationalitäten überlagert, die selbst Vermischungen von Werbung und Information, Marktmacht und Profit kaschieren:

- In technischer Hinsicht lässt sich feststellen, dass sich Inhalte-Anbieter reaktiv bei der Gestaltung ihrer Seiten zunehmend stärker an den Relevanzkriterien von Suchmaschinen orientieren, auch lenkende, intervenierende Metadaten setzen und damit die vorgebliche Neutralität, Sachlichkeit und Relevanzmaxime der Suche konterkarieren, mithin die Trefferauswahl in ihrem Interesse steuern (Röhle 2010, 163).

- Marktstrategisch ist Werbung inzwischen zum Kerngeschäft des Unternehmens geworden - nicht nur wie anfangs ausschließlich durch kontextsensitive Werbung, sondern durch die Übernahme von Doubleclicks heute mit allen Varianten der Online-Werbung: nämlich mit Display Ads, Bookmarks- und Affiliate Marketing. Gegenüber der Werbevermarktung geraten Suchfunktion und andere Dienstleistungen ins Hintertreffen, der User wird durch die möglichst exakte Zuordnung von Werbebotschaften zum Konsumenten moduliert (Ebd., 187).

- Verschiedene Tracking-Verfahren und behavioural, ja personalisiertes Targeting der User ermöglichen es Google, Daten unterschiedlichster Art zu erfassen und miteinander in Beziehung zu setzen. Damit werden nicht nur äußert präzise Daten von Tätigkeiten, Präferenzen, Konsumbedürfnissen zu Profilen von Usern generiert, vielmehr ergeben sich intensive Potentiale der Aufmerksamkeitsmessung und -steuerung, die auch politisch und gesellschaftlich verwendet werden können und ein immenses Risiko der Überwachung und des Missbrauchs darstellen (Ebd., 203ff; Pariser 2012).

Außerdem scheint der Weltmonopolist seine Systeme zunehmend zu schließen und dem ehedem propagierten Prinzip der Offenheit entgegenzuarbeiten: Funktionen und Dienste werden ständig erweitert, so dass der User alles, was er/sie braucht, im Google-Kosmos vorfindet. Immer mehr Seiten weisen seit etwa 2010 wiederum auf Google-Seiten, so dass auch hier lukrative Zyklen generiert werden. So wirft der Wettbewerbskommissar der EU, Joaquin Almunia, im Frühjahr 2012 Google folgende Praktiken vor: 
- Erstens verknüpft Google generelle Suchfragen mit eigenen, weiterführenden Suchmaschinen, die leichter zu erkennen sind als die der Konkurrenz und auf der Ergebnisseite die oberen Plätze belegen. Verbraucher werden so von der Nutzung anderer Anbieter abgehalten.

- Zweitens missachtet Google den Schutz geistigen Eigentums, weil es beispielsweise Erfahrungsberichte von Internetnutzern kopiert und ohne Autorisierung auf seine eigenen Seiten stellt.

- Als dritten Punkt führt die Behörde an, dass Google Werbepartner gezwungen hat, auf ihren Seiten keine anderen Suchmaschinen zu platzieren. Diese Praxis hat Folgen für Online-Shops oder Online-Magazine.

- Viertens hat Google die Übertragbarkeit von Werbung auf seiner eigenen Plattform AdWords auf Seiten der Konkurrenz verhindert, indem SoftwareEntwickler vertraglich am Angebot solcher Übertragungsprogramme gehindert wurden (Bolzen 2012).

Wie auch bei den Offerten anderer Konzerne sind die inzwischen massiv verbreiteten Apps nicht zuletzt dafür geeicht, fremde, nicht im jeweiligen System vermarktbare Apps zu blockieren und auf die eigene Versionen hinzuführen ( $\mathrm{Wu}$ 2012, 346f). Apples i-Geräte, nämlich iPod, iPhone und iPad, formieren solche Restriktionen sogar in technischer Hinsicht, gewissermaßen als Hardwareleistungen. Zwar sind alle theoretisch universal nutzbare Computer, aber unter Marketinggesichtspunkten und der Maxime der Benutzerfreundlichkeit sind sie auf spezielle Funktionen kapriziert, so dass sich ein diversifizierter Markt und ein entsprechendes Kaufverhalten ergibt: iPod für das optimale Abspielen von Videos und Musik, iPhone als Handy, Internetanschluss, Kamera und zusätzliche Funktionen für unterwegs, iPad als das mobile Desktop, allerdings mit eingeschränkter Bedienungsfreundlichkeit für aktive Nutzung und Produktion (Wu 2012, 339f). Solche Ausdifferenzierungen sind - im Grunde genommen - gegenüber der universalen, integrativen Technik des Computers kontraproduktiv; mit einschlägigem Knowhow können User sie umgehen und unterlaufen. Sie bestätigen allein Konsum und Prestigedenken des durchschnittlichen Kunden, der mit Besitz und Nutzung mehrere Geräte sein finanzielles Potential und seine Fortschrittlichkeit unterstreichen will. Insofern setzen solche Technik- und Marktkonditionierungen durch die Anbieter gewissermaßen eine technische Internet Governance, da Nutzung und Wahrnehmung des Internets durch sie geprägt werden - mindestens prätendiert wird, es so zu verstehen und zu nutzen.

Eine fatale Kombination aus Profilmodulation und Personalisierung, eine so genannte „Filter Bubble“, sehen Kritiker (Pariser 2012; Meckel 2011) am Werk, 
vor allem bei den IT-Größen wie Google, Amazon und Facebook. Mit jedem Click feilen und komplettieren Cookies, Crawler und andere Hilfsprogramme am jeweiligen Nutzerporträt, so dass jede/r nur noch die Daten und Angebote präsentiert bekommt, die seinen/ihren üblichen Gewohnheiten und Vorlieben entsprechen. Das mag einerseits bequem und hilfreich sein, weil aus der ungeheuren Fülle der Daten nur noch die erwünschten und gebräuchlichen ausgewählt, mithin die Masse und Komplexität des Datenkosmos auf erträgliches Maß reduziert werden. Doch mit ihnen schrumpfen auch die Optionen von Überraschung, Neuigkeit, Ungewöhnlichem: „Obwohl das Internet Zugang zu einem überwältigenden Spektrum von Quellen und Optionen bietet, bekommen wir [...] in der Filter Bubble nur wenig davon mit. Obwohl das Internet uns neue Möglichkeiten gibt, unsere Identitäten auszubilden oder auszuprobieren, drängt die Ökonomie der Personalisierung zu einer statischen Auffassung von Persönlichkeit. Obwohl das Internet das Potential zur Dezentralisierung von Wissen und Macht hat, konzentriert es die Kontrolle darüber, was wir gezeigt bekommen und welche Möglichkeiten wir geboten bekommen, und legt die Macht in die Hände von immer weniger Personen" (Pariser 2011; 228).

So dürften digitale Informationen und der mediale Alltag an der Oberfläche immer üppiger und differenzierter ausfallen. Und sicherlich lassen sich präformierte Gewichtungen und Restriktionen immer wieder aufbrechen und umgehen, mit entsprechender Absicht und Kompetenz. Doch zugleich dürften sich unauffällige, kaum mehr bewusste Gewohnheiten als Engführungen und Einseitigkeiten einschleifen, die kaum mehr die Offenheit und Pluralität vorhalten, die ehedem dem Internet bescheinigt wurde - so wie Google mittlerweile weltweit die überragend genutzte, fast exklusive Suchmaschine geworden ist: „Die Serverfarmen der Googles und Amazons werden wachsen, während die Prozessoren in ihnen schrumpfen, und diese enorme Rechenleistung wird genutzt, um immer genauere Vorhersagen über unsere Vorlieben oder gar unser Seelenleben zu treffen. Personalisierte Technologien der ,erweiterten Realität` werden unsere Wahrnehmung der realen Welt immer mehr überlagern" (Pariser 2011, 200). Auch wenn die Mediengeschichte und ihre Bewertung voll von solchen kulturkritischen Übertreibungen ist, bleibt doch zu bedenken, dass es neben expliziten Regelungen gewissermaßen vielfältige latente Tendenzen gibt, Internet Governance zumal von privater Seite als technische, programmierte und damit auf jeden Einzelnen speziell abgestimmte, vor allem für den Markt lukrative zu lancieren und auf diese Weise explizite, mühsam auszuhandelnde, öffentliche Regelungen zu unterlaufen oder gar zu ersetzen. 